\title{
Surgical reconstruction of leprotic foot-drop
}

\author{
M. M. BARI, * A. K. M. S. ISLAM \\ \& A. K. M. A. HAQUE \\ Reconstructive and Orthopaedic Surgery Unit, Leprosy Control \\ Institute and Hospital, Mohakhali, Dhaka, Bangladesh
}

\section{Accepted for publication 14 November 1995}

\begin{abstract}
Summary We have operated on 25 patients for correction of foot-drop due to leprosy from March 1992 to July 1994. The method used was circumtibial transfer of the tibialis posterior to the tendons of extensor hellucis longus and the extensor digitorum longus in the foot together with lengthening of the Achilles tendon. The results were satisfactory in 20 of these cases as judged by adequate restoration of heel-toe gait and of active dorsiflexion. The follow-up period ranged from 6 months to 2 years. Inadequate post-operative physiotherapy was the reason for unsatisfactory results in five cases.
\end{abstract}

\section{Introduction}

It is well recognized that correction of foot-drop deformity in leprosy is essential to ensure normal walking and to prevent plantar ulcerations. This paper describes the procedure adopted and the results of surgical correction of foot-drop due to leprosy in 25 patients at the Leprosy Control Institute and Hospital, Mohakhali, Dhaka in Bangladesh. The procedure adopted was the transfer of the tendon of the tibialis posterior muscle to the extensor tendons of the toes in the foot so as to ensure active dorsiflexion of the foot. ${ }^{1-3}$

\section{Material and methods}

In the Institute between March 1992 and July 1994 we saw 34 patients with foot-drop due to leprosy. Nine of these were treated conservatively and 25 were treated surgically. Of these 25 patients 20 were male and 5 female. Their ages ranged from 12 to 45 years. Contracture of the Achilles tendon was present in all cases. The follow-up period was 6 months to 2 years.

* Correspondence: Dr Md Mofakhkharul Bari, 3/10-GBlock, Lalmatia, Dhaka, Bangladesh. 
PRE-OPERATIVE FINDINGS

The duration of foot-drop because of paralysis of the lateral popliteal nerve was at least one year in all cases. Contracture of the Achilles tendon was present in all cases. The foot-at-rest angle ranged from 103 to 122 degrees the average being 111.5 degrees.

\section{PRE-OPERATIVE PHYSIOTHERAPY}

It is essential to isolate the action of the muscle to be transferred and to ensure that its strength is adequate. This was done by teaching the patient to first learn isolated action of the tibialis posterior muscle and then to exercise against resistance.

\section{OPERATION PROCEDURE}

Lengthening of the Achilles tendon was done in all cases as a first step. The tibialis posterior tendon was servered near its distal attachment to the navicular bone, withdrawn into the leg, split longitudinally into two slips and both slips transferred circumtibially to the front of the foot. Keeping the ankle in 70 degrees of dorsiflexion and the foot in neutral version, one slip was sutured to the tendon of extensor hellucis longus and the other to the tendons of the extensor digitorum longus. After closure of wounds the limb was immobilized in a Plaster of Paris (POP) boot for 6 weeks.

\section{POST-OPERATIVE PHYSICAL THERAPY}

Initially the lower limb was elevated using a Bohler-Braun frame. Weight-bearing was allowed after 4 days and the patient discharged. Six weeks after the operation the patient was readmitted, POP and sutures removed and a strong posterior slab was given to rest the limb with ankle in full dorsiflexion except during therapy. Isolated contraction of the transferred muscle by exercises similar to the pre-operative ones was now started in reclining position. Exercises in dependent-foot position were started as soon as the patient could learn to contract the transferred muscle.

Partial weight-bearing was started in the second week. This is the crucial period when the patient progresses from active contraction of the transfer without weight-bearing on the foot to gradually increased weight-bearing and relearning the heel-toe walking pattern. Initially walking is done between parallel bars. This training is continued till the 4th week, by then the patient is able to achieve a good heel-toe gait with full weight being borne on the foot. Except for the therapy period the limb is immobilized in the posterior slab for the rest of the day. Without proper physiotherapy the operation fails.

\section{Results}

In 20 out of 25 patients active dorsiflexion of $77 \cdot 6$ degrees on average was achieved as well as a satisfactory heel-toe gait. The result was hence considered satisfactory. The range of active dorsiflexion was found to be 80 to 72 degrees (10 to 18 degrees above 90 degrees) while the range of active movement at the ankle was 15 to 20 degrees in these 20 cases. In 5 cases the result was poor because of inadequate physiotherapy postoperatively. The active dorsiflexion in these cases was nil in one case and inadequate 
in others, while none could walk with a heel-toe gait. They were advised to repeat the operation.

\section{Discussion}

The aims in correction of foot-drop are attainment of active dorsiflexion of the foot above 90 degrees and restoration of the heel-toe gait. We have used the transfer of the tibialis posterior to the toe tendons as our method of choice. In 20 of 25 cases we have obtained good results, adequate dorsiflexion of the foot and restoration of the heel-toe gait. In our opinion when the foot can be dorsiflexed above the neutral position a good result is obtained. Adequate training by physiotherapy before and after surgery is essential for success. We have found this method of treatment of foot-drop to be excellent. Self-care and proper protective foot-wear is essential to keep the foot free of ulcers.

\section{References}

1 Fritchi EP. Values and limitation of surgery in leprosy. Lepr. India, 1976; 48 (1): 47.

2 Fritchi EP. Surgical reconstruction and rehabilitation in leprosy. New Delhi 1984, p. 166-76.

${ }^{3}$ Weber MW, Van Soest A, Neff G, Chiang T \& Pfau R. Results of surgical procedure for the correction of foot drop and lagophthalmus due to leprosy. Lepr Rev, 1992; 63: 255-62. 